\title{
Analysis and Simulation of SIRS Model for Dengue Fever Transmission in South Sulawesi, Indonesia
}

\author{
Wahidah Sanusi, ${ }^{1}$ Nasiah Badwi, ${ }^{2}$ Ahmad Zaki, ${ }^{1}$ Sahlan Sidjara, ${ }^{1}$ Nurwahidah Sari, \\ Muhammad Isbar Pratama, ${ }^{1}$ and Syafruddin Side $(10)^{1}$ \\ ${ }^{1}$ Mathematics Department, Universitas Negeri Makassar, Makassar 90222, Indonesia \\ ${ }^{2}$ Geography Department, Universitas Negeri Makassar, Makassar 90222, Indonesia \\ Correspondence should be addressed to Syafruddin Side; syafruddin@unm.ac.id
}

Received 1 August 2020; Revised 11 December 2020; Accepted 31 December 2020; Published 15 January 2021

Academic Editor: Yansheng Liu

Copyright ( 2021 Wahidah Sanusi et al. This is an open access article distributed under the Creative Commons Attribution License, which permits unrestricted use, distribution, and reproduction in any medium, provided the original work is properly cited.

\begin{abstract}
This study is aimed at building and analysing a SIRS model and also simulating the model to predict the number of dengue fever cases. Methods applied for this model are building the SIRS model by modifying the SIR model, analysing the SIRS model using the Lyapunov function to prove three theorems (the existence, the free disease, and the endemic status of dengue fever), and simulating the SIRS model using the number of dengue case data in South Sulawesi by Maple. The results obtained are the SIRS model of dengue fever transmission, stability analysis, global stability, and the value of the basic reproduction number $R_{0}$. The simulation done for the dengue fever case in South Sulawesi found the basic reproduction number $R_{0}=26.47609>1$; it means that South Sulawesi is in the endemic stage of transmission for dengue fever disease. Simulation of the SIRS model for dengue fever can predict the number of dengue cases in South Sulawesi that could be a recommendation for the government in an effort to prevent the number of dengue fever cases.
\end{abstract}

\section{Introduction}

The World Health Organization [1] revealed the rapid increase in the number of dengue fever (DF) cases that reached 30 times during the last 50 years. Considered the most transmitting disease in the world, the DF virus may potentially infect more than half of the world population. Annual data reports [1] show that the number of patients suffering from the DF in hospitals is in the interval of a half to a million people.

Dengue fever (DF) is a type of disease in tropical regions, especially Indonesia, including South Sulawesi [1]. Based on data from the Ministry of Health of the Republic of Indonesia (2013), the number of dengue cases in South Sulawesi has increased from year to year as shown in Figure 1. The following is the updated DF victims as of 20 January 2016 for several districts/cities in South Sulawesi province: 32 city cases of Makassar, 645 cases of Bone district, 11 cases of Gowa district, 56 cases of Maros district, 125 cases of Pangkep dis- trict, 23 cases of Sinjai district, and 225 cases of Luwu district, while other districts/cities have not been recorded [1].

Studies investigating appropriate mathematical models for the dengue fever cases were done using the model of Suspected, Infected, and Removed (SIR) and Suspected, Exposed, Infected, and Removed (SEIR) [2-21]. The SIR models assumed that individuals who recovered from the disease would no longer be infected. Recent facts, however, show possibilities of a recovered patient to be suspected for the second time. This becomes the main reason to modify the SIR into SIRS [19]. This paper is the extension of the SIRS model [19] with analysis, and the simulation presented in this article is a reliable alternative reference to control the happening of DF in a region. The former part of the article discusses formulation of the SIRS model for the DF transmission, the second part contains analysis of the model involving three theorems and investigation of the model stability, and the last part presents simulations of the SIRS model performed for both free disease and endemic cases. 


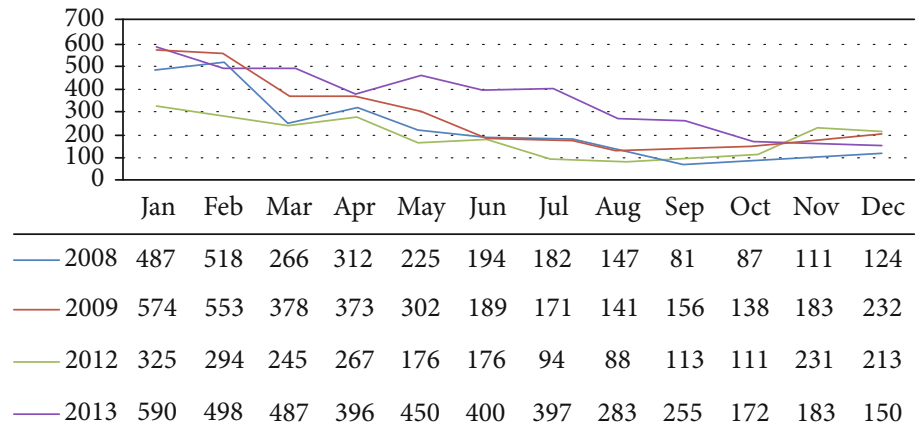

Figure 1: Trends in dengue fever cases in South Sulawesi.

\section{Materials and Methods}

This is a theoretical study on the model of dengue fever transmission. The SIRS model is developed by modifying the SIR model $[8,9]$ that has been previously used. The model is then analysed to prove the existence theorem, the free disease, and the endemic cases of DF using the method of Lyapunov function $[10,11]$. Afterwards, global stability of the SIRS is investigated using the Eigenvalue equation [9]. The simulation of the model used the number of dengue case data in South Sulawesi by Maple. The simulation is done to describe and predict the number of dengue fever cases in South Sulawesi.

\section{Results}

3.1. SIRS Model of Dengue Fever Disease Transmission. There are several assumptions used in model formation: the total population of humans and mosquitoes is considered constant, the rate of birth and mortality rate are considered equal, births in mosquitoes and human populations in each class enter into the suspected class, each individual in the population is likely to have the same mosquito bites, the infected mosquito bite rate is higher than the suspected mosquito, and each recovered individual has the possibility of reinfection so that it reenters the suspected class. The parameters used in the dengue fever disease model are presented in Table 1.

Changes that occur in the human and the mosquito classes can be interpreted in Figure 2.

Figure 2 can be interpreted in the form of a mathematical model that is a host-vector interaction model which is the following nonlinear differential equation:

$$
\begin{aligned}
& \frac{d S_{h}}{d t}=\mu_{h} N_{h}-\frac{\beta_{h} b}{N_{h}} I_{v} S_{h}-\mu_{h} S_{h}+\theta_{h} R_{h} \\
& \frac{d I_{h}}{d t}=\frac{\beta_{h} b}{N_{h}} I_{v} S_{h}-\left(\mu_{h}+\gamma_{h}\right) I_{h} \\
& \frac{d R_{h}}{d t}=\gamma_{h} I_{h}-\left(\mu_{h}+\theta_{h}\right) R_{h} \\
& \frac{d S_{v}}{d t}=\mu_{v} N_{v}-\frac{\beta_{v} b}{N_{h}} I_{h} S_{v}-\mu_{v} S_{v} \\
& \frac{d I_{v}}{d t}=\frac{\beta_{v} b}{N_{h}} I_{h} S_{v}-\mu_{v} I_{v}
\end{aligned}
$$

with conditions $S_{h}+I_{h}+R_{h} \leq N_{h}$ and $N_{v}=A / \mu_{v}$.
TABle 1: Definition of variable/parameter.

\begin{tabular}{lc}
\hline Variable/parameter & Definition \\
\hline$N_{h}$ & Number of human population \\
$N_{v}$ & Number of mosquito population \\
$\mu_{h}$ & Birth and mortality of human population \\
$\mu_{v}$ & Birth and mortality of mosquito population \\
$b$ & The rate of mosquito bites \\
$\gamma_{h}$ & The rate of cure for disease \\
$\theta_{h}$ & The rate of decline in human immunity to \\
$\beta_{v}$ & Probability of spreading virus from $I_{h}$ to $S_{v}$ \\
$\beta_{h}$ & Probability of spreading virus from $I_{v}$ to $S_{h}$ \\
$\beta_{v} b$ & Interaction capabilities $I_{h}$ and $S_{v}$ \\
$\beta_{h} b$ & Interaction capabilities $I_{v}$ and $S_{h}$ \\
$A$ & The rate of mosquito recruitment \\
\hline
\end{tabular}

\section{Analysis of SIRS Model for Dengue Fever Transmission}

All variables and parameters in the model are nonnegatives as observable in the equation system (1), and the nonnegative octant $R_{+}^{5}$ is positive invariant. Based on the equation system (1), we derive Theorem 1; we shall prove the positivity of solution for the SIRS model.

Theorem 1. Let $\left(S_{h}(t)>0, I_{h}(t)>0, R_{h}(t)>0, S_{v}(t)>0, I_{v}\right.$ $(t)>0)$ be the solution of the equation system (1) with initial condition $\left(S_{0 h}, I_{0 h} \cdot R_{0 h}, S_{0 v}, I_{0 v}\right)$ on the compact set

$$
\begin{aligned}
D= & \left\{\left(S_{h}(t), I_{h}(t), R_{h}(t), S_{v}(t), I_{h}(t)\right) \in R_{+}^{5}, L_{1}\right. \\
& \left.=S_{h}+I_{h}+R_{h} \leq N_{h}, L_{2}=S_{v}+I_{v} \leq N_{v}=\frac{A}{\mu_{v}}\right\} .
\end{aligned}
$$

For the model system (1), the region $D$ is a positive invariant that contains any solutions of $R_{+}^{5}$. 


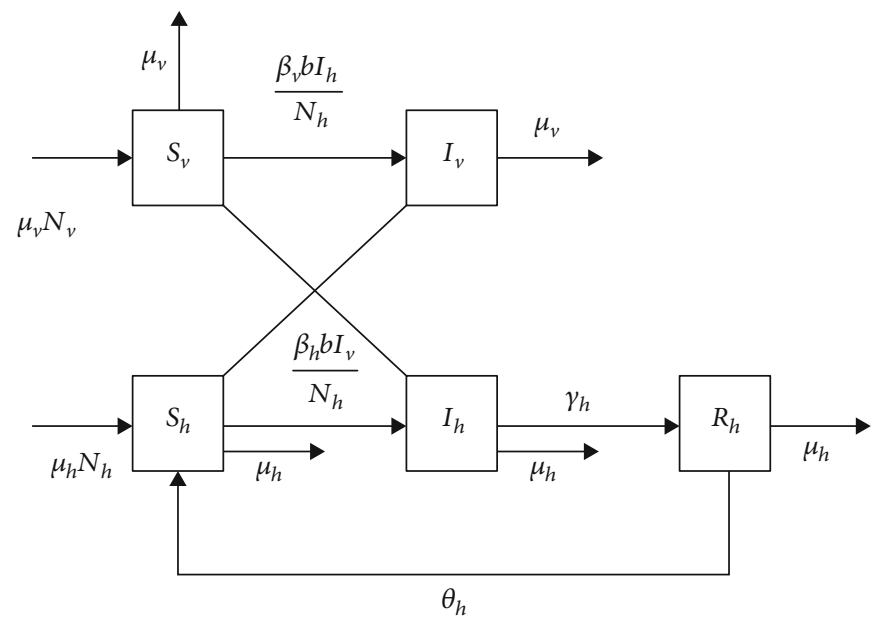

FIGURE 2: SIRS model diagram of human and vector population [19].

Proof. A Lyapunov function constructed for the system is

$$
L(t)=\left(L_{1}(t), L_{2}(t)\right)=\left(S_{h}+I_{h}+R_{h}, S_{v}+I_{v}\right) .
$$

The derivative of $L(t)$ with respect to time that satisfies equation (3) is

$$
\begin{aligned}
\frac{d L}{d t}= & \left(\frac{d l_{1}}{d t}, \frac{d L_{2}}{d t}\right)=\left(\frac{d S_{h}}{d t}+\frac{d I_{h}}{d t}+\frac{d R_{h}}{d t}, \frac{d S_{v}}{d t}+\frac{d I_{v}}{d t}\right) \\
= & \mu_{h} N_{h}-\frac{\beta_{h} b I_{h}}{N_{h}} S_{h}-\mu_{h} S_{h}+\theta_{h} R_{h}+\frac{\beta_{h} b}{N_{h}} I_{v} S_{h} \\
& -\left(\mu_{h}+\gamma_{h}\right) I_{h}+\gamma_{h} I_{h}-\left(\mu_{h}+\theta_{h}\right) R_{h}, \mu_{v} N_{v} \\
& -\frac{\beta_{v} b}{N_{h}} I_{h} S_{v}-\mu_{v} S_{v}+\frac{\beta_{v} b}{N_{h}} I_{v} S_{v}-\mu_{v} I_{v} \\
= & \left(\mu_{h} N_{h}-\mu_{h}\left(S_{h}+I_{h}+R_{h}\right), A-\mu_{v}\left(S_{v}+I_{v}\right)\right) \\
= & \left(\mu_{h} N_{h}-\mu_{h} L_{1}, A-\mu_{v} L_{2}\right) .
\end{aligned}
$$

Thus, we can find

$$
\left\{\begin{array}{l}
\frac{d L_{1}}{d t}=\mu_{h} N_{h}-\mu_{h} L_{1} \leq 0, \text { for } L_{1} \geq N_{h} \\
\frac{d L_{2}}{d t}=A-\mu_{v} L_{2} \leq 0, \text { for } L_{2} \geq \frac{A}{\mu_{v}}
\end{array}\right.
$$

Hence, by equation (5), we obtained that $d L / d t \leq 0$ which implies that $D$ is a positive invariant set. Meanwhile, solving equation system (5) results in

$$
0 \leq\left(L_{1}(t), L_{2}(t)\right) \leq\left(N_{h}+L_{1}(0) e^{-\mu_{h} t}, \frac{A}{\mu_{v}}+L_{2}(0) e^{-\mu_{v} t}\right),
$$

where $L_{1}(0)$ and $L_{2}(0)$ are the initial condition of $L_{1}(t)$ and $L_{2}(t)$ consecutively.

Hence, as $t \rightarrow \infty, 0 \leq\left(L_{1}(t), L_{2}(t)\right) \leq\left(N_{h}, A / \mu_{h}\right)$. This confirms that $D$ is a positive invariant set containing all the solutions in $R_{+}^{5}$. This proves Theorem 1 .
Theorem 1 guarantees the existence of DF transmission in a region in which the DF transmitting virus was formerly absent and then changed when the population of suspected but not infected, $S_{h}(t)>0$, infected, $I_{h}(t)>0$, and recovered individual, $R_{h}(t)>0$, from DF was found. The theorem also indicates an advance study on the stages of the DF transmission by which a region can be identified as free disease or endemic with the use of the SIRS model.

\section{Global Stability Analysis}

System (1) for the SIRS model of the DF transmission applies the equilibrium value of $\left(S_{h}^{*}, I_{h}^{*}, R_{h}^{*}, S_{v}^{*}, I_{v}^{*}\right)=\left(N_{h}, 0,0, A / \mu_{v}, 0\right)$. The Jacobian matrix of equation system (1) is defined as follows:

$J=\left[\begin{array}{ccccc}-\frac{\beta_{h} b I_{v}}{N_{h}}-\mu_{h} & 0 & \theta_{h} & 0 & -\frac{\beta_{h} b S_{h}}{N_{h}} \\ \frac{\beta_{h} b I_{v}}{N_{h}} & -\mu_{h}-\gamma_{h} & 0 & 0 & \frac{\beta_{h} b S_{h}}{N_{h}} \\ 0 & \gamma_{h} & -\mu_{h}-\theta_{h} & 0 & 0 \\ 0 & -\frac{\beta_{v} b S_{v}}{N_{h}} & 0 & -\frac{\beta_{v} b I_{h}}{N_{h}}-\mu_{v} & 0 \\ 0 & \frac{\beta_{v} b S_{v}}{N_{h}} & 0 & \frac{\beta_{v} b I_{h}}{N_{h}} & -\mu_{v}\end{array}\right]$.

By considering the equilibrium point, we obtain

$$
J=\left[\begin{array}{ccccc}
-\mu_{h} & 0 & \theta_{h} & 0 & -\beta_{h} b \\
0 & -\mu_{h}-\gamma_{h} & 0 & 0 & \beta_{h} b \\
0 & \gamma_{h} & -\mu_{h}-\theta_{h} & 0 & 0 \\
0 & -\frac{\beta_{v} b S_{v}}{N_{h}} & 0 & -\mu_{v} & 0 \\
0 & \frac{\beta_{v} b S_{v}}{N_{h}} & 0 & 0 & -\mu_{v}
\end{array}\right]
$$


In order to find the Eigenvalue $\lambda$, we simplify system (1) and then solve the equation $|J-\lambda I|$, that is,

$$
\begin{aligned}
& \left|\left[\begin{array}{ccccc}
-\mu_{h}-\lambda & 0 & \theta_{h} & 0 & -\beta_{h} b \\
0 & -\mu_{h}-\gamma_{h}-\lambda & 0 & 0 & \beta_{h} b \\
0 & \gamma_{h} & -\mu_{h}-\theta_{h}-\lambda & 0 & 0 \\
0 & -\frac{\beta_{v} b S_{v}}{N_{h}} & 0 & -\mu_{v}-\lambda & 0 \\
0 & \frac{\beta_{v} b S_{v}}{N_{h}} & 0 & 0 & -\mu_{v}-\lambda
\end{array}\right]\right|=0 \text {, } \\
& \left(-\mu_{h}-\lambda\right)\left|\left[\begin{array}{cccc}
-\mu_{h}-\gamma_{h}-\lambda & 0 & 0 & \beta_{h} b \\
\gamma_{h} & -\mu_{h}-\theta_{h}-\lambda & 0 & 0 \\
-\frac{\beta_{v} b S_{v}}{N_{h}} & 0 & -\mu_{v}-\lambda & 0 \\
\frac{\beta_{v} b S_{v}}{N_{h}} & 0 & 0 & -\mu_{v}-\lambda
\end{array}\right]\right|=0 \text {, } \\
& \begin{aligned}
&\left(-\mu_{h}-\lambda\right)\left(-\mu_{v}-\lambda\right)\left(-\mu_{h}-\theta_{h}-\lambda\right) \\
& \cdot\left(\left(-\mu_{h}-\gamma_{h}-\lambda\right)\left(-\mu_{v}-\lambda\right)-\left(\beta_{h} b\right) \beta_{v} b\right)=0
\end{aligned}
\end{aligned}
$$

The Eigenvalues obtained are

$$
\begin{aligned}
& \lambda_{1}=-\mu_{h}, \\
& \lambda_{2}=-\mu_{v}, \\
& \lambda_{3}=-\mu_{h}-\theta_{h},
\end{aligned}
$$

and the Eigenvalue equation is

$$
\lambda^{2}+\left(\mu_{h}+\mu_{v}+\gamma_{h}\right) \lambda+\left(\mu_{h}+\gamma_{h}\right) \mu_{v}-b \beta_{h} b \beta_{v}=0
$$

From equation (11), the basic reproduction number $R_{0}$ for system (1) of the SIRS model can be obtained using the method $[20,21]$, that is,

$$
R_{0}=\frac{b \beta_{h} b \beta_{v}}{\mu_{v}\left(\mu_{h}+\gamma_{h}\right)} .
$$

\section{Global Stability of Free Disease Equilibrium of Model SIRS}

Equation system (1) applies free disease equilibrium of $\left(S_{h}^{*}\right.$, $\left.I_{h}^{*}, R_{h}^{*}, S_{v}^{*}, I_{v}^{*}\right)=\left(N_{h}, 0,0, A / \mu_{v}, 0\right)$ indicating the possibility of the disease to fade out. Theorem 2 explains the behavior of the free disease equilibrium globally for equation (1).

Theorem 2. If $R_{0} \leq 1$, then the free disease equilibrium $\left(S_{h}^{*}\right.$, $\left.I_{h}^{*}, R_{h}^{*}, S_{v}^{*}, I_{v}^{*}\right)=\left(N_{h}, 0,0, A / \mu_{v}, 0\right)$ in the global stage is asymptotically stable in $D$, by assuming that

$$
\begin{aligned}
& \mu_{h}=\frac{\beta_{v} b}{N_{h}} S_{v}^{*}, \\
& \mu_{v}=\frac{\beta_{h} b}{N_{h}} S_{h}^{*} .
\end{aligned}
$$

Proof. A Lyapunov function constructed for the system is

$$
W(t)=\left(S_{h}-S_{h}^{*} \ln S_{h}\right)+I_{h}+R_{h}+\left(S_{v}-S_{v}^{*} \ln S_{v}\right)+I_{v} .
$$

The derivative of $W(t)$ with respect to time that satisfies equation (14) is

$$
\begin{aligned}
& \dot{W}(t)=\dot{S_{h}}\left(1-\frac{S_{h}^{*}}{S_{h}}\right)+\dot{I}_{h}+\dot{R}_{h}+\dot{S_{v}}\left(1-\frac{S_{v}^{*}}{S_{v}}\right)+\dot{I}_{v} \\
& =\left(\mu_{h} N_{h}-\frac{\beta_{h} b I_{v}}{N_{h}} S_{h}-\mu_{h} S_{h}+\theta_{h} R_{h}\right)\left(1-\frac{S_{h}^{*}}{S_{h}}\right) \\
& +\frac{\beta_{h} b I_{v}}{N_{h}} S_{h}-\mu_{h} I_{h}-\gamma_{h} I_{h}+\gamma_{h} I_{h}-\mu_{h} R_{h}-\theta_{h} R_{h} \\
& +\left(A-\frac{\beta_{v} b I_{h}}{N_{h}} S_{v}-\mu_{v} S_{v}\right)\left(1-\frac{S_{v}^{*}}{S_{v}}\right)+\frac{\beta_{v} b I_{v}}{N_{h}} S_{v}-\mu_{v} I_{v} \\
& =\mu_{h} N_{h}\left(1-\frac{S_{h}^{*}}{S_{h}}\right)+\mu_{h} S_{h}^{*}\left(1-\frac{S_{h}}{S_{h}^{*}}\right)-\frac{\beta_{h} b I_{v}}{N_{h}} S_{h} \\
& +\frac{\beta_{h} b I_{v}}{N_{h}} S_{h}\left(\frac{S_{h}^{*}}{S_{h}}\right)-\theta_{h} R_{h}\left(\frac{S_{h}^{*}}{S_{h}}\right)+\frac{\beta_{h} b I_{v}}{N_{h}} S_{h}-\mu_{h} I_{h} \\
& -\mu_{h} R_{h}+A\left(1-\frac{S_{v}^{*}}{S_{v}}\right)+\mu_{v} S_{v}^{*}\left(1-\frac{S_{v}}{S_{v}^{*}}\right)-\frac{\beta_{v} b I_{h}}{N_{h}} S_{v} \\
& +\frac{\beta_{v} b I_{h}}{N_{h}} S_{v}\left(\frac{S_{v}^{*}}{S_{v}}\right)+\frac{\beta_{v} b I_{v}}{N_{h}} S_{v}-\mu_{v} I_{v} \\
& =\mu_{h} N_{h}\left(1-\frac{S_{h}^{*}}{S_{h}}\right)+\mu_{h} S_{h}^{*}\left(1-\frac{S_{h}}{S_{h}^{*}}\right)+\frac{\beta_{h} b I_{v}}{N_{h}} S_{h}^{*} \\
& -\theta_{h} R_{h}\left(\frac{S_{h}^{*}}{S_{h}}\right)-\mu_{h} I_{h}-\mu_{h} R_{h}+A\left(1-\frac{S_{v}^{*}}{S_{v}}\right) \\
& +\mu_{v} S_{v}^{*}\left(1-\frac{S_{v}}{S_{v}^{*}}\right)+\frac{\beta_{v} b I_{h}}{N_{h}} S_{v}^{*}-\mu_{v} I_{v} \\
& =\mu_{h} N_{h}\left(1-\frac{S_{h}^{*}}{S_{h}}\right)+\mu_{h} S_{h}^{*}\left(1-\frac{S_{h}}{S_{h}^{*}}\right)-\theta_{h} R_{h}\left(\frac{S_{h}^{*}}{S_{h}}\right) \\
& -\mu_{h} R_{h}+A\left(1-\frac{S_{v}^{*}}{S_{v}}\right)+\mu_{v} S_{v}^{*}\left(1-\frac{S_{v}}{S_{v}^{*}}\right) \\
& +\left(\frac{\beta_{v} b}{N_{h}} S_{v}^{*}-\mu_{h}\right) I_{h}+\left(\frac{\beta_{h} b}{N_{h}} S_{h}^{*}-\mu_{v}\right) I_{v} .
\end{aligned}
$$

Considering $S_{h}^{*}=N_{h}, S_{v}^{*}=A / \mu_{v}$, condition (13) to equation (15) can be expressed as

$$
\begin{aligned}
\dot{W}(t)= & \mu_{h} N_{h}\left(1-\frac{S_{h}^{*}}{S_{h}}+1-\frac{S_{h}}{S_{h}^{*}}\right)-\theta_{h} R_{h}\left(\frac{S_{h}^{*}}{S_{h}}\right)-\mu_{h} R_{h} \\
& +A\left(1-\frac{S_{v}^{*}}{S_{v}}+1-\frac{S_{v}}{S_{v}^{*}}\right)=\mu_{h} N_{h}\left(2-\frac{S_{h}^{*}}{S_{h}}-\frac{S_{h}}{S_{h}^{*}}\right) \\
& -\theta_{h} R_{h}\left(\frac{S_{h}^{*}}{S_{h}}\right)-\mu_{h} R_{h}+A\left(2-\frac{S_{v}^{*}}{S_{v}}-\frac{S_{v}}{S_{v}^{*}}\right) \\
= & -\mu_{h} N_{h} \frac{\left(S_{h}-S_{h}^{*}\right)^{2}}{S_{h} S_{h}^{*}}-\theta_{h} R_{h}\left(\frac{S_{h}^{*}}{S_{h}}\right)-A \frac{\left(S_{v}-S_{v}^{*}\right)^{2}}{S_{v} S_{v}^{*}}-\mu_{h} R_{h} .
\end{aligned}
$$


Equation (16) shows that $\dot{W}(t) \leq 0$. Using the Lyapunov method [10], the finite sets applicable for the solution are those contained in the largest invariant set, where $S_{h}=S_{h}^{*}$, $R_{h}=0$, and $S_{v}=S_{v}^{*}$, that is, the singleton set $\left\{S_{h}^{*}, I_{h}^{*}, R_{h}^{*}, S_{v}^{*}\right.$, $\left.I_{v}^{*}\right\}$. This implies if the free disease equilibrium $S_{h}^{*}, I_{h}^{*}, R_{h}^{*}$, $S_{v}^{*}, I_{v}^{*}$ is the global stage asymptotically stable in $D$. This proves Theorem 2 .

This global stability theorem for the free disease case of the SIRS model explains a stage of the existence of the DF case, as explained in Theorem 1 . Theorem 2 says that if $R_{0}$ $\leq 1$, then an infected individual will not infect others. Thus, DF in this stage can still be controlled and should not be worried about.

\section{Global Stability of Endemic Equilibrium of Model SIRS}

The SIRS model of system (1) has an equilibrium point of $P^{* *}=\left(S_{h}^{* *}, I_{h}^{*}, R_{h}^{* *}, S_{v}^{* *}, I_{v}^{* *}\right) \in D$ called the endemic equilibrium point, which satisfies $S_{h}^{* *}>0, I_{h}^{* *}>0, R_{h}^{* *}>0, S_{v}^{* *}>0$, $I_{v}^{* *}>0$, where

$$
\begin{aligned}
& S_{h}^{* *}=\frac{N_{h}^{2} \mu_{v}\left(\mu_{h}+\gamma_{h}\right)\left(\beta_{v} \mu_{h} b+\beta_{v} b \theta_{h}+\mu_{h} \mu_{v}+\mu_{v} \gamma_{h}+\mu_{v} \theta_{h}\right)}{\left(\beta_{h} \mu_{h} A b+\beta_{h} A b \gamma_{h}+\beta_{h} A b \theta_{h}+\mu_{h} \mu_{v} N_{h}+\mu_{h} \mu_{v} N_{h} \gamma_{h}+\mu_{h} \mu_{v} N_{h} \theta_{h}+\mu_{v} N_{h} \gamma_{h} \theta_{h}\right) \beta_{v} b}, \\
& I_{h}^{* *}=\frac{\left(\mu_{h}+\theta_{h}\right) N_{h}\left(\beta_{h} \beta_{v} A b^{2}-\mu_{h} \mu_{v}^{2} N_{h}-\mu_{v}^{2} N_{h} \gamma_{h}\right)}{\left(\beta_{h} \mu_{h} A b+\beta_{h} A b \gamma_{h}+\beta_{h} A b \theta_{h}+\mu_{h}^{2} \mu_{v} N_{h}+\mu_{h} \mu_{v} N_{h} \gamma_{h}+\mu_{h} \mu_{v} N_{h} \theta_{h}+\mu_{v} N_{h} \gamma_{h} \theta_{h}\right) \beta_{v} b}, \\
& R_{h}^{* *}=\frac{N_{h} \gamma_{h}\left(\beta_{h} \beta_{v} A b^{2}-\mu_{h} \mu_{v}^{2} N_{h}-\mu_{v}^{2} N_{h} \gamma_{h}\right)}{\left(\mu_{h} A b+\beta_{h} A b \gamma_{h}+\beta_{h} A b \theta_{h}+\mu_{h}^{2} \mu_{v} N_{h}+\mu_{h} \mu_{v} N_{h} \gamma_{h}+\mu_{h} \mu_{v} N_{h} \theta_{h}+\mu_{v} N_{h} \gamma_{h} \theta_{h}\right) \beta_{v} b}, \\
& S_{v}^{* *}=\frac{\beta_{h} \mu_{h} A b+\beta_{h} A b \gamma_{h}+\beta_{h} A b \theta_{h}+\mu_{h}^{2} \mu_{v} N_{h}+\mu_{h} \mu_{v} N_{h} \gamma_{h}+\mu_{h} \mu_{v} N_{h} \theta_{h}+\mu_{v} N_{h} \gamma_{h} \theta_{h}}{\left(\beta_{v} \mu_{h} b+\beta_{v} b \theta_{h}+\mu_{h} \mu_{v}+\mu_{v} \gamma_{h}+\mu_{v} \theta_{h}\right) \beta_{h} b}, \\
& I_{v}^{* *}=\frac{\left(\mu_{h}+\theta_{h}\right)\left(\beta_{h} \beta_{v} A b^{2}-\mu_{h} \mu_{v}^{2} N_{h}-\mu_{v}^{2} N_{h} \gamma_{h}\right)}{\mu_{v}\left(\beta_{v} \mu_{h} b+\beta_{v} b \theta_{h}+\mu_{h} \mu_{v}+\mu_{v} \gamma_{h}+\mu_{v} \theta_{h}\right) \beta_{h} b} .
\end{aligned}
$$

The following theorem explains the endemic global equilibrium of system (1).

Theorem 3. If $R_{0}>1$, then the equilibrium status of $D F$ diseases is positively endemic, and equation system (1) exists and is in the global stage asymptotically stable in D by assuming that

$$
\begin{gathered}
S_{h}^{* *}=N_{h}, \\
S_{v}^{* *}=\frac{A}{\mu_{v}}, \\
\mu_{h}=\frac{\beta_{v} b}{N_{h}} \frac{\left(\mu_{h}+\gamma_{h}+\theta_{h}\right)}{r}, \\
\mu_{v}=\frac{r \beta_{h} b}{\left(\mu_{h}+\gamma_{h}+\theta_{h}\right)} S_{v}^{* *},
\end{gathered}
$$

where $r=\beta_{h} b / N_{h}, \mu_{v}$ is the rate of mosquito population mortality, $N_{h}$ is the number of the human population which is likely the same as the number of DF suspected, $b$ is the rate of potentially infecting mosquito bites, and $\beta_{h} b$ is the interaction capability between humans and mosquitoes as the vector.

Proof. We constructed the Lyapunov function of the form in

$$
\begin{aligned}
V(t)= & \left(S_{h}-S_{h}^{* *} \ln S_{h}\right)+I_{h}+R_{h}+\frac{\mu_{h}+\gamma_{h}+\theta_{h}}{r S_{v}^{* *}}\left(S_{v}-S_{v}^{* *} \ln S_{v}\right) \\
& +\frac{\mu_{h}+\gamma_{h}+\theta_{h}}{r S_{v}^{* *}} I_{v} .
\end{aligned}
$$

The derivative of $V(t)$ with respect to time that satisfies equation (19) is

$$
\begin{aligned}
& \dot{V}(t)=\dot{S}_{h}\left(1-\frac{S_{h}^{* *}}{S_{h}}\right)+\dot{I}_{h}+\dot{R}_{h}+\dot{S}_{v}\left(1-\frac{S_{v}^{* *}}{S_{v}}\right)\left(\frac{\mu_{h}+\gamma_{h}+\theta_{h}}{r S_{v}^{* *}}\right) \\
& +\frac{\mu_{h}+\gamma_{h}+\varphi_{h}}{r S_{v}^{* *}} \dot{I}_{v}=\mu_{h} N_{h}\left(1-\frac{S_{h}^{* *}}{S_{h}}\right)+\theta_{h} R_{h}\left(1-\frac{S_{h}^{* *}}{S_{h}}\right) \\
& -\mu_{h} S_{h}\left(1-\frac{S_{h}^{* *}}{S_{h}}\right)-\frac{b \beta_{h} I_{v}}{N_{h}}\left(1-\frac{S_{h}^{* *}}{S_{h}}\right)+\frac{b \beta_{h} I_{v}}{N_{h}} S_{h} \\
& -\mu_{h} I_{h}-\gamma_{h} I_{h}+\gamma_{h} I_{h}-\mu_{h} R_{h}-\theta_{h} R_{h}+\left(\frac{\mu_{h}+\gamma_{h}+\theta_{h}}{r S_{v}^{* *}}\right) \\
& \cdot A\left(1-\frac{S_{v}^{* *}}{S_{v}}\right)-\left(\frac{\mu_{h}+\gamma_{h}+\theta_{h}}{r S_{v}^{* *}}\right) \frac{b \beta_{v} I_{h}}{N_{h}} S_{v}\left(1-\frac{S_{v}^{* *}}{S_{v}}\right) \\
& -\left(\frac{\mu_{h}+\gamma_{h}+\theta_{h}}{r S_{v}^{* *}}\right) \mu_{v} S_{v}\left(1-\frac{S_{v}^{* *}}{S_{v}}\right)+\left(\frac{\mu_{h}+\gamma_{h}+\theta_{h}}{r S_{v}^{* *}}\right) \\
& \cdot \frac{b \beta_{v} I_{h}}{N_{h}} S_{v}-\left(\frac{\mu_{h}+\gamma_{h}+\theta_{h}}{r S_{v}^{* *}}\right) \mu_{v} I_{v}=\mu_{h} N_{h}\left(1-\frac{S_{h}^{* *}}{S_{h}}\right) \\
& +\theta_{h} R_{h}-\theta_{h} R_{h}\left(\frac{S_{h}^{* *}}{S_{h}}\right)-\mu_{h} S_{h}\left(1-\frac{S_{h}^{* *}}{S_{h}}\right)-\frac{b \beta_{h} I_{v}}{N_{h}} S_{h} \\
& +\frac{b \beta_{h} I_{v}}{N_{h}} S_{h}^{* *}+\frac{b \beta_{h} I_{v}}{N_{h}} S_{h}-\mu_{h} I_{h}-\mu_{h} R_{h}-\theta_{h} R_{h} \\
& +A\left(\frac{\mu_{h}+\gamma_{h}+\theta_{h}}{r S_{v}^{* *}}\right)-A\left(\frac{\mu_{h}+\gamma_{h}+\theta_{h}}{r S_{v}}\right)-\frac{b \beta_{v} I_{h}}{N_{h}} S_{v} \\
& \cdot\left(\frac{\mu_{h}+\gamma_{h}+\theta_{h}}{r S_{v}^{* *}}\right)+\frac{b \beta_{v} I_{h}}{N_{h}}\left(\frac{\mu_{h}+\gamma_{h}+\theta_{h}}{r}\right) \\
& -\left(\frac{\mu_{h}+\gamma_{h}+\theta_{h}}{r}\right) \mu_{v}\left(\frac{S_{v}}{S_{v}^{* *}}\right)+\mu_{v}\left(\frac{\mu_{h}+\gamma_{h}+\theta_{h}}{r}\right) \\
& +\left(\frac{\mu_{h}+\gamma_{h}+\theta_{h}}{r}\right) \frac{b \beta_{h} I_{v}}{N_{h}}\left(\frac{S_{v}}{S_{v}^{* *}}\right)-\left(\frac{\mu_{h}+\gamma_{h}+\theta_{h}}{r S_{\mathrm{v}}^{* *}}\right) \mu_{v} I_{v} \\
& =\mu_{h} N_{h}\left(1-\frac{S_{h}^{* *}}{S_{h}}\right)+\mu_{h} S_{h}^{* *}\left(1-\frac{S_{h}^{* *}}{S_{h}}\right)-\theta_{h} R_{h}\left(\frac{S_{h}^{* *}}{S_{h}}\right)-\mu_{h} R_{h} \\
& +\frac{b \beta_{h} I_{v}}{N_{h}} S_{h}^{* *}-\mu_{h} I_{h}+A\left(\frac{\mu_{h}+\gamma_{h}+\theta_{h}}{r S_{v}^{* *}}\right)-A\left(\frac{\mu_{h}+\gamma_{h}+\theta_{h}}{r S_{v}}\right) \\
& +\frac{b \beta_{v} I_{h}}{N_{h}}\left(\frac{\mu_{h}+\gamma_{h}+\theta_{h}}{r}\right)-\left(\frac{\mu_{h}+\gamma_{h}+\theta_{h}}{r}\right) \mu_{v}\left(\frac{S_{v}}{S_{v}^{* *}}\right) \\
& +\mu_{v}\left(\frac{\mu_{h}+\gamma_{h}+\theta_{h}}{r}\right)-\left(\frac{\mu_{h}+\gamma_{h}+\theta_{h}}{r S_{v}^{* *}}\right) \mu_{v} I_{v} \text {. }
\end{aligned}
$$


Substituting equation (18) into equation (20), we can find

$$
\begin{aligned}
\dot{V(t)=} & \mu_{h} N_{h}\left[2-\frac{S_{h}^{* *}}{S_{h}}-\frac{S_{h}}{S_{h}^{* *}}\right]-\theta_{h} R_{h}\left(\frac{S_{h}^{* *}}{S_{h}}\right) \\
& -\mu_{h} R_{h}+\left(\frac{b \beta_{h}}{N_{h}} S_{h}^{* *}-\left(\frac{\mu_{h}+\gamma_{h}+\theta_{h}}{r S_{v}^{* *}}\right) \mu_{v}\right) I_{v} \\
& +\left(\frac{b \beta_{v}}{N_{h}}\left(\frac{\mu_{h}+\gamma_{h}+\theta_{h}}{r}\right)-\mu_{h}\right) I_{h}+\left(\frac{\mu_{h}+\gamma_{h}+\theta_{h}}{r S_{v}^{* *}}\right) \mu_{v} S_{v}^{* *} \\
& -\left(\frac{\mu_{h}+\gamma_{h}+\theta_{h}}{r S_{V}}\right) \mu_{v} S_{v}^{* *}+\mu_{v}\left(\frac{\mu_{h}+\gamma_{h}+\theta_{h}}{r}\right) \\
& -\left(\frac{\mu_{h}+\gamma_{h}+\theta_{h}}{r}\right) \mu_{v}\left(\frac{S_{v}}{S_{v}^{* *}}\right),
\end{aligned}
$$

$$
\begin{aligned}
\mu_{h} N_{h}[2 & \left.-\frac{S_{h}^{* *}}{S_{h}}-\frac{S_{h}}{S_{h}^{* *}}\right]-\theta_{h} R_{h}\left(\frac{S_{h}^{* *}}{S_{h}}\right)-\mu_{h} R_{h}+\frac{2 \mu_{v}}{r}\left(\mu_{h}+\gamma_{h}+\theta_{h}\right) \\
& -\left(\frac{\mu_{h}+\gamma_{h}+\theta_{h}}{r}\right) \mu_{v}\left(\frac{S_{v}}{S_{v}^{* *}}\right)-\left(\frac{\mu_{h}+\gamma_{h}+\theta_{h}}{r}\right) \mu_{v}\left(\frac{S_{v}^{* *}}{S_{v}}\right) \\
& \cdot \mu_{h} N_{h}\left[2-\frac{S_{h}^{* *}}{S_{h}}-\frac{S_{h}}{S_{h}^{* *}}\right]+\left(\frac{\mu_{h}+\gamma_{h}+\theta_{h}}{r}\right) \mu_{v}\left[2-\frac{S_{v}^{* *}}{S_{v}}-\frac{S_{v}}{S_{v}^{* *}}\right] \\
& -\theta_{h} R_{h}\left(\frac{S_{h}^{* *}}{S_{h}}\right)-\mu_{h} R_{h}=-\mu_{h} N_{h}\left[\frac{\left(S_{h}-S_{h}^{* *}\right)^{2}}{S_{h} S_{h}^{* *}}\right] \\
& -\mu_{v}\left[\frac{\mu_{h}+\gamma_{h}+\theta_{h}}{r}\right]\left[\frac{\left(S_{v}-S_{v}^{* *}\right)^{2}}{S_{v} S_{v}^{* *}}\right]-\theta_{h} R_{h}\left(\frac{S_{h}^{* *}}{S_{h}}\right)-\mu_{h} R_{h} .
\end{aligned}
$$

Equation (22) shows that $\dot{V}(t) \leq 0$ for all $S_{h}^{* *}, I_{h}^{*}, R_{h}^{* *}$, $S_{v}^{* *}, I_{v}^{* *} \in D, S_{h}^{* *}, I_{h}^{* *}, R_{h}^{* *}, S_{v}^{* *}, I_{v}^{* *}$ and $\dot{V}(t)=0$ for $S_{h}=S_{h}^{* *}, I_{h}$ $=I_{h}^{* *}, R_{h}=R_{h}^{* *}, S_{v}=S_{v}^{* *}$, and $I_{v}=I_{v}^{* *}$. Equilibrium $P^{*}$ is a set of positive invariant of system (1) that is contained in

$$
\begin{aligned}
L=\{ & \left(S_{h}(t), I_{h}(t), R_{h}(t), S_{v}(t), I_{v}(t)\right), S_{h}=S_{h}^{* *}, I_{h}=I_{h}^{* *}, \\
& \left.R_{h}=R_{h}^{* *}, S_{v}=S_{v}^{* *}, I_{v}=I_{v}^{* *}\right\} .
\end{aligned}
$$

Using the asymptotical stability theorem, positive endemic equilibrium $P^{*}$ is in the global stage asymptotically stable in $D$. This proves Theorem 3 .

The global stability theorem for the model SIRS in this stage tells if an individual is infected with DF with $R_{0}>1$; then, the individual will likely infect at least another individual. Thus, the DF in this situation has been endemic, uncontrolled, and threatening for the human population within the region.

\section{Simulation of SIRS Model for Dengue Fever Transmission in South Sulawesi, Indonesia}

Simulation of the model is performed using the software Maple. Initial values $S_{h}(0), I_{h}(0), R_{h}(0), S_{v}(0)$, and $I_{v}(0)$ used in the simulation are based on the data shown in Table 2, which are data related to dengue fever in South Sulawesi. Meanwhile, the basic reproduction number $R_{0}$ is obtained from equation (12), that is,
TABLE 2: The initial and parameter values of the SIRS model simulation.

\begin{tabular}{lccccc}
\hline Variable & $\begin{array}{c}\text { Initial } \\
\text { values }\end{array}$ & Source & Parameter & $\begin{array}{c}\text { Value } \\
\left(R_{0}>1\right)\end{array}$ & Source \\
\hline$N_{h}(0)$ & 8771970 & {$[22]$} & $b \beta_{h}$ & 0.750000 & {$[9]$} \\
$S_{h}(0)$ & 8768197 & {$[22]$} & $\mu_{h}$ & 0.000046 & {$[9]$} \\
$I_{h}(0)$ & 1895 & {$[23]$} & $\theta_{h}$ & 0.575000 & Assumption \\
$R_{h}(0)$ & 1878 & {$[23]$} & $\gamma_{h}$ & 0.328833 & {$[9]$} \\
$N_{v}(0)$ & 1000000 & {$[9]$} & $\mu_{v}$ & 0.032300 & {$[9]$} \\
$S_{v}(0)$ & 944000 & {$[9]$} & $b \beta_{v}$ & 0.375000 & {$[9]$} \\
$I_{v}(0)$ & 56000 & {$[9]$} & & & \\
\hline
\end{tabular}

$$
R_{0}=\frac{b \beta_{v} b \beta_{h}}{\mu_{v}\left(\mu_{h}+\gamma_{h}\right)} .
$$

\section{Stability Analysis of Model SIRS for Dengue Fever in South Sulawesi}

Stability analysis of the obtained equilibrium values is determined by the Eigenvalues $\lambda$ [9]. Based on equations (10) and (11), Eigenvalues of the SIRS for the transmission of DF are obtained. The stability of the system, categorized in types as in [20], depends on the Eigenvalues. Model stability analysis of the SIRS model with the initial value in Table 2 is as follows.

The critical value is determined by substituting parameter values of the free disease cases from Table 2. Assuming that equation system (1) is zero, equation system (25) is found as follows:

$$
\begin{gathered}
0.000046-0.0855 I_{v} S_{h}-0.000046 S_{h}+0.575 R_{h}=0, \\
0.0855 I_{v} S_{h}-0.328879 I_{h}=0, \\
0.328833 I_{h}-0.575046 R_{h}=0 \\
0.0323-0.375 I_{h} S_{v}-0.0323 S_{v}=0, \\
0.375 I_{h} S_{v}-0.0323 I_{v}=0 .
\end{gathered}
$$

The Eigenvalues from equations (10) and (11) with parameters described in Table 2 for this SIRS model of the DF transmission are

$$
\begin{gathered}
\lambda_{1}=-0.000046, \\
\lambda_{2}=-0.0323, \\
\lambda_{3}=-0.575064, \\
\lambda_{4}=-0.328995, \\
\lambda_{5}=-0.032184 .
\end{gathered}
$$

The obtained Eigenvalues $\lambda$ are real and negatives. Thus, according to [20], the stability of this equilibrium point is asymptotically stable in South Sulawesi. The basic reproduction number using the number of dengue fever in South 


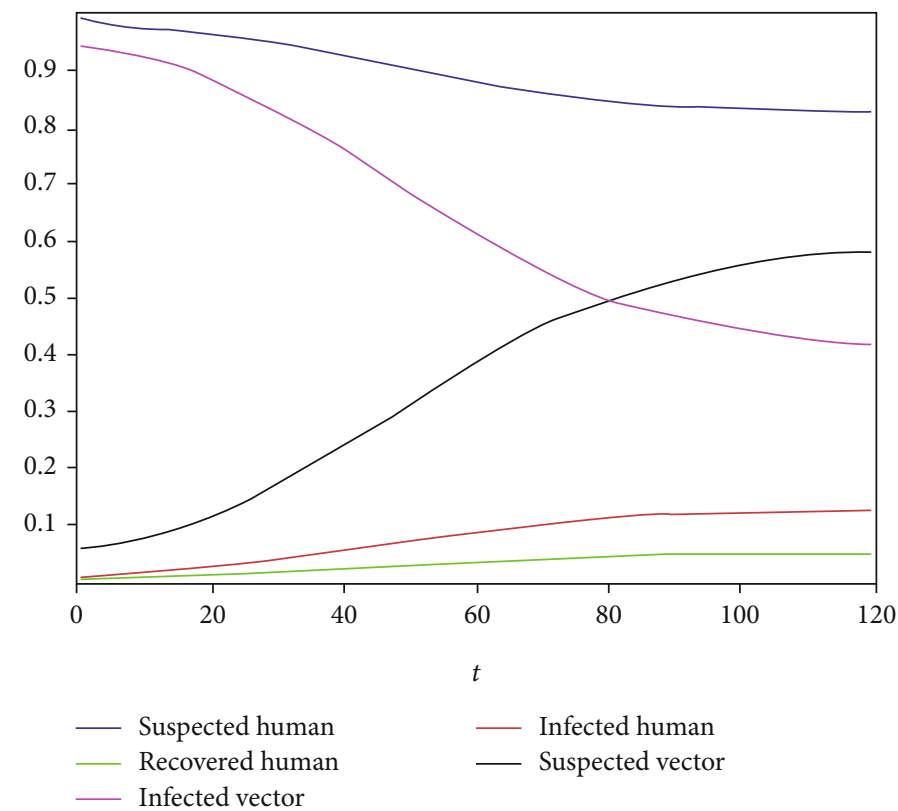

FIGURE 3: Suspected, infected, and recovered prediction of dengue fever in South Sulawesi.

Sulawesi is $R_{0}=26.47609>1$, which suggests if an infected individual will infect up to 26 others. That means South Sulawesi is in the endemic stage of dengue fever.

\section{Simulation Result of Model SIRS for DF Transmission in South Sulawesi}

Simulation results of the SIRS model toward DF transmissions obtained using the software Maple with initial values and parameters, described in Table 2, are shown in Figure 2 . The values are given on the $x$-axis, while the $y$-axis shows time (in month) of each of the variables $S_{h}(t), I_{h}(t)$, $R_{h}(t), S_{v}(t)$, and $I_{v}(t)$.

The result of the SIRS model shows if DF transmission requires only very short time to diminish. Based on Figure 3, the number of dengue fever cases (infected humans) in South Sulawesi reaches its peak immediately in six years; meanwhile, the number of suspected humans in South Sulawesi is very high and needs a long time for the decrease. Meanwhile, the number of infected mosquitoes declines four months after its maximum number. Thus, the mosquito population can be reduced quickly in South Sulawesi, and this region becomes endemic to dengue fever. This corresponded to the basic reproduction number value in South Sulawesi $R_{0}=26.47609>1$ and according to Theorem 3; thus, South Sulawesi is endemic to dengue fever.

\section{Discussion}

Studies of the mathematical model of the transmission of dengue fever have been performed by [10], producing the SIR model with analysis using Lyapunov function. In addition, [9] did a simulation of the SIR using data from South Sulawesi, Indonesia, and Selangor, Malaysia. The numerical solution of the SIR model for the transmission of DF has been investigated by [8] using the homotopy perturbation method, then [18], producing a SEIRS model with analysis using the Lyapunov function.

This article produces the SIRS model as a modification of the SIR [19], given the fact that recovered individuals from DF will still have a possibility to be suspected. The result of this paper given the analysis of the SIRS is performed using three theorems, namely, the existence, free disease, and endemic DF using the method of Lyapunov function. Simulation results using data from the Health Ministry of the Republic of Indonesia show that South Sulawesi is an endemic area of dengue fever; then, early control and prevention measures need to be done because the climate in Indonesia is increasingly difficult to predict, especially the rainy season which causes many larvae of Aedes mosquitoes spreading dengue virus. The simulation result also can predict the number of suspected, infected, and recovered DF cases in South Sulawesi in the coming months.

\section{Conclusion}

Based on the result and the discussion, the SIRS model for the transmission of DF is obtained. The three theorems produced suggest the existence of DF in a region, free disease status of DF, and the endemic status. The resulted basic reproduction number $R_{0}$ is able to predict the possible infected population from an infected DF patient. The simulation result done is able to predict the number of DF cases in South Sulawesi, and the basic reproduction number is $R_{0}=$ $26.47609>1$; thus, South Sulawesi is in the endemic stage of dengue fever. The simulation result also as such can be used as a reference to prevent and control the number of dengue fever cases in South Sulawesi. 


\section{Data Availability}

The data used to finding support from this paper are available from related author papers as well as online data.

\section{Conflicts of Interest}

The authors declare that they have no conflicts of interest.

\section{Acknowledgments}

We would like to thank UNM No: 558/UN36.11/HK/2020 for the financial support.

\section{References}

[1] WHO, "Fact sheets: dengue and dengue haemorrhagic fever," 2015, June 2016, http://www.who.int/mediacentre/factsheets/ fs $117 /$ en/, Tribun timur.

[2] M. Derouich and A. Boutayeb, "Dengue fever: mathematical modelling and computer simulation," Applied Mathematics and Computation, vol. 177, no. 2, pp. 528-544, 2006.

[3] L. Esteva and C. Vargas, "Analysis of a dengue disease transmission model," Mathematical Biosciences, vol. 150, no. 2, pp. 131-151, 1998.

[4] A. Korobeikov, "Lyapunov functions and global properties for SEIR and SEIS epidemic models," Mathematical Medicine and Biology, vol. 21, pp. 75-83, 2014.

[5] G. Li, W. Wang, and Z. Jin, "Global stability of an SEIR epidemic model with constant immigration," Chaos, Solitons \& Fractals, vol. 30, no. 4, pp. 1012-1019, 2006.

[6] N. Nuraini, E. Soewono, and K. Sidarto, "Mathematical model of dengue disease ransmission with severe dhf compartment," Bulletin of the Malaysian Mathematical Sciences Society, vol. 30, no. 2, pp. 143-157, 2007.

[7] P. Pongsumpun, "Transmission model for dengue disease with and without the effect of extrinsic incubation period," KMITL Sci. Tech., vol. 6, pp. 74-82, 2006.

[8] Y. M. Rangkuti, S. Side, and M. S. M. Noorani, "Numerical analytic solution of SIR model of dengue fever disease in South Sulawesi using homotopy perturbation method and Variational iteration method," Journal of Mathematical and Fundamental Sciences., vol. 46, no. 1, pp. 91-105, 2014.

[9] S. Side and S. M. Noorani, "A SIR model for spread of dengue fever disease (simulation for South Sulawesi, Indonesia and Selangor, Malaysia)," World Journal of Modelling and Simulation, vol. 9, no. 2, pp. 96-105, 2013.

[10] S. Syafruddin and M. S. M. Noorani, "Lyapunov function of SIR and SEIR model for transmission of dengue fever disease," International Journal of Simulation and Process Modelling, vol. 8, no. 2/3, pp. 177-184, 2013.

[11] S. Side, W. Sanusi, M. K. Aidid, and S. Sidjara, "Global stability of SIR and SEIR model for tuberculosis disease transmission with Lyapunov function method," Asian Journal of Applied Sciences, vol. 9, no. 3, pp. 87-96, 2016.

[12] E. Soewono and A. K. Supriatna, "A two-dimensional model for the transmission of dengue fever disease," Bulletin of the Malaysian Mathematical Sciences Society, vol. 24, pp. 49-57, 2005.
[13] A. K. Supriatna and E. Soewono, "Model Matematika Penyebaran Penyakit Demam berdarah," Jurnal Bionatura, vol. 2, no. 3, pp. 104-116, 2000.

[14] S. M. O’Regan, T. C. Kelly, A. Korobeinikov, M. J. A. O’Callaghan, and A. V. Pokrovskii, "Lyapunov functions for SIR and SIRS epidemic models," Applied Mathematics Letters, vol. 23, no. 4, pp. 446-448, 2010.

[15] Y. Yaacob, "Analysis of a dengue disease transmission model without immunity," Matematika, vol. 23, no. 2, pp. 75-81, 2007.

[16] G. Z. Zeng, L. S. Chen, and L. H. Sun, "Complexity of an SIR epidemic dynamics model with impulsive vaccination control," Chaos, Solitons \& Fractals, vol. 26, no. 2, pp. 495-505, 2005.

[17] K. C. Mozhgan and R. Paul, "Simulating heterogeneous behaviours in complex systems on GPUs," Simulation Modelling Practice and Theory, vol. 83, pp. 3-17, 2018.

[18] W. Nur, H. Rachman, N. M. Abdal, M. Abdy, and S. Side, "SIR Model Analysis for Transmission of Dengue Fever Disease with Climate Factors Using Lyapunov Function," Journal of Physics, vol. 1028, no. 1, Article 012117, 2018.

[19] S. Side, A. Zaki, and N. Sari, "A SIRS model for transmission of dengue fever disease in South Sulawesi," in International Conferences on Mathematics and Natural Scinces, pp. 81-90, Harris Sunset Road, Kuta Bali, Indonesia, 2017.

[20] S. Side, W. Sanusi, N. Badwi et al., "A SEIRS model analysis and simulation for dengue fever transmission," International Journal of Scientific \& Technology Research, vol. 8, no. 10, pp. 1048-1053, 2019.

[21] O. Diekmann and J. O. P. Hesterbeek, Mathematical Epidemiology of Infectious Diseases, John Wliley, Chichester, 2000.

[22] O. Diekmann, J. A. Heesterbeek, and J. A. J. Metz, "On the definition and the computation of the basic reproduction ratio $\mathrm{R} 0$ in models for infectious diseases in heterogeneous populations," Journal of Mathematical Biology, vol. 28, no. 4, pp. 365382, 1990.

[23] BPS, "Jumlah Penduduk Sulawesi Selatan," 2018, November 2019, https://tumoutounews.com/2018/04/11/jumlahpenduduk-sulawesi-selatan-terbaru-tahun-2018/. 